\section{CONSUMO ALIMENTAR DE VITAMINA A POR GESTANTES NO BRASIL: UMA REVISÃO SISTEMÁTICA}

\author{
Vitamin "A" food consumption by pregnant women in Brazil: \\ a systematic review
} Consumo alimentario de vitamina A en embarazadas de Brasil:
una revisión sistemática

\section{RESUMO}

Objetivo: Realizar uma revisão sistemática sobre o consumo alimentar de vitamina A por gestantes no Brasil. Métodos: A revisão constituiu na busca de artigos publicados no período de 1999 a 2015 nas bases SciELO, PubMed e LILACS. Ao final, foram selecionados oito artigos para esta revisão. Resultados: Os métodos utilizados para análise do consumo de vitamina A foram o questionário de frequência alimentar (QFA) - da dieta ou apenas de alimentos fontes de vitamina A - e o recordatório alimentar (R24h). Apenas dois artigos calcularam a adequação da ingestão de vitamina A para a população avaliada. Algumas limitações metodológicas foram bastante frequentes, destacando-se a ausência e/ou limitação de informações sobre representatividade da amostra, perdas do estudo, acurácia dos métodos aplicados e controle das variáveis de confusão. Conclusão: Observa-se que ainda são escassos os estudos que avaliam de forma criteriosa o consumo de vitamina A por gestantes no Brasil, e que a identificação e controle dos possíveis vieses do inquérito dietético poderão melhorar a fidedignidade dos dados encontrados.

Descritores: Deficiência de Vitamina A; Consumo Alimentar; Ingestão de Alimentos; Vitamina A; Gravidez.

\section{ABSTRACT}

Objective: To conduct a systematic review of the Vitamin " $A$ " food consumption by pregnant women in Brazil. Methods: The review consisted of a search for articles published in the period from 1999 to 2015 in SciELO, PubMed, and LILACS databases. At the end, eight articles were selected for this review. Results: The methods used for the analysis of the intake of vitamin " $A$ " were: food frequency questionnaire (FFQ) - considering the diet or only vitamin " $A$ " foods and the dietary recall (24hDR). Only two articles estimated the adequacy of the Vitamin " $A$ " food consumption by the population assessed. Some methodological limitations were quite frequent, emphasizing the lack and/or limitation of information on the sample representativeness, loss of studies, accuracy of the methods applied and the control of confounding variables. Conclusion: It is observed that there are still few studies that critically assess the Vitamin "A" food consumption by pregnant women in Brazil, and that the identification and control of possible biases of the dietary surveys can improve the reliability of the information found.

Drescriptors: Vitamin A Deficiency; Food consumption; Food Intake; Vitamin A; Pregnancy.
Artigo de Revisão

1) Universidade Federal do Piauí - UFPI Teresina (PI) - Brasil

Recebido em: 28/08/2015 Revisado em: $10 / 09 / 2015$ Aceito em: 29/09/2015 


\section{RESUMEN}

Objetivo: Realizar una revisión sistemática sobre el consumo alimentario de vitamina A en embarazadas de Brasil. Métodos: La revisión se dio por la búsqueda de artículos publicados en el periodo entre 1999 y 2015 en las bases de datos SciELO, PubMed y LILACS. Por fin fueron seleccionados ocho artículos para esta revisión. Resultados: Los métodos utilizados para el análisis del consumo de la vitamina A fueron el cuestionario de la frecuencia alimentaria (QFA) - de la dieta o solamente de alimentos fuentes de vitamina $A-y$ el recordatorio de $24 h$ (R24h). Solamente dos artículos calcularon la adecuación para ingestión de vitamina $A$ para la población evaluada. Algunas limitaciones metodológicas fueron muy frecuentes, destacándose la ausencia y/o limitación de informaciones sobre la representatividad de la muestra, las pérdidas del estudio, la eficacia de los métodos aplicados y el control de las variables de confusión. Conclusión: Todavía se observan pocos estudios que evalúan con criterios el consumo de la vitamina A en las embarazadas de Brasil y que la identificación y el control de los posibles sesgos de la averiguación dietética puede que mejore la fidedignidad de los datos encontrados.

Descriptores: Deficiencia de Vitamina A; Consumo de Alimentos; Ingestión de Alimentos; Vitamina A; Embarazo.

\section{INTRODUÇÃO}

A vitamina A é um nutriente essencial, importante na promoção do crescimento e desenvolvimento, e ainda na manutenção da função imune e de reprodução ${ }^{(1,2)}$, com papel de destaque em períodos de intenso crescimento proliferativo e desenvolvimento tecidual, como os observados na gestação e desenvolvimento fetal ${ }^{(3)}$.

A deficiência de vitamina A (DVA) encontra-se entre as grandes prioridades de carência de micronutrientes, afetando cerca de 210 milhões de crianças menores de cinco anos, gestantes e lactantes em todo mundo ${ }^{(2,4)}$. No Brasil, um dos países mais afetados da América, dados da última Pesquisa Nacional de Demografia e Saúde da Criança e da Mulher (PNDS) $)^{(5)}$, realizada em 2006, indicaram que 12,3\% das mulheres apresentaram níveis inadequados de vitamina A, com os maiores índices de inadequação encontrados no Nordeste $(19,0 \%)$ e no Sudeste $(21,6 \%)$ do país.

ADVAé frequente na gestação e compromete o resultado do processo gravídico em diversos aspectos, contribuindo para infecções ${ }^{(6)}$, prematuridade ${ }^{(7)}$, baixo peso ao nascer ${ }^{(8)}$, anemia $^{(9)}$, más-formações ${ }^{(10)}$ e síndromes hipertensivas da gravidez $^{(11)}$, entretanto, os seus efeitos sobre a mortalidade materna e infantil ainda são inconclusivos ${ }^{(12-14)}$. Ela tem como principal causa a baixa ingestão de alimentos fontes ${ }^{(2)}$, mas também pode estar associada a outros fatores, como os socioeconômicos e o saneamento básico inadequado ${ }^{(15)}$.
Tendo em vista a complexidade e o alto custo de pesquisas em grande escala para estimar a prevalência de deficiência de vitamina A mediante indicadores bioquímicos, o inquérito alimentar pode ser uma ferramenta valiosa para avaliação do estado nutricional dos indivíduos. A escolha do método depende da população a ser estudada e do objetivo do estudo ${ }^{(16)}$.

Nas gestantes, as alterações do estado fisiológico e psicológico podem influenciar os resultados dos estudos de análise do consumo alimentar. Os métodos que melhor se aplicam a estudos epidemiológicos são o recordatório alimentar de 24 horas (R24h), a história dietética (HD) e a frequência de consumo alimentar (QFA), todos de caráter retrospectivo ${ }^{(17)}$. Porém, a utilização de instrumentos de avaliação do consumo, quando não segue uma metodologia de aplicação criteriosa, gera resultados não reprodutíveis. No Brasil, poucos estudos abordaram o consumo de vitamina A por gestantes. Nesse sentido, objetivou-se realizar uma revisão sistemática sobre o consumo alimentar de vitamina A por gestantes no Brasil.

\section{MÉTODOS}

No presente estudo, foi adotado o método de revisão sistemática da literatura, consistindo na busca retrospectiva de artigos científicos, neste caso, sobre as pesquisas realizadas no Brasil e publicadas em âmbito nacional ou internacional que analisaram o consumo de vitamina A em gestantes, através de inquéritos dietéticos.

Os artigos foram identificados por meio de busca nas seguintes bases de dados: SciELO (Scientific Electronic Library Online), LILACS (Literatura Latino-Americana e do Caribe em Ciências da Saúde) e PubMed (National Library of Medicine, Bethesda, MD). Os descritores utilizados foram: "(deficiência de vitamina A) AND (consumo alimentar OR ingestão de alimentos) AND (vitamina A) AND (gravidez)" e seus correspondentes em inglês e espanhol, conforme a terminologia adotada pelo sítio dos Descritores em Ciências e Saúde - DeCS. O período de publicação dessa revisão foi de 1999 a 2015. $\mathrm{O}$ vasto intervalo de tempo utilizado para abrangência dos artigos foi devido à escassez de estudos que tenham avaliado o consumo de vitamina A por gestantes no país, bem como à ausência de levantamentos nacionais objetivando a mesma temática a que se propõe o presente estudo.

A busca foi realizada de forma independente por três examinadores, conforme os seguintes critérios de elegibilidade: estudos com desenhos do tipo coorte, casocontrole e transversal que tenham sido publicados em português, inglês e espanhol. 
A seleção dos estudos ocorreu em três etapas. Inicialmente, por meio da análise dos títulos dos artigos, os examinadores avaliaram aqueles identificados pelas combinações dos descritores nas bases de dados propostas. Em seguida, após seleção dos títulos relevantes, procedeu-se à leitura dos resumos. Nos casos em que houve necessidade de maiores informações, o texto foi explorado na íntegra, para posteriormente ser julgado pertinente ao tema de estudo e, em seguida, analisado.

Os artigos foram avaliados em relação aos aspectos metodológicos e científicos, os quais foram estabelecidos com base em uma escala ${ }^{(18)}$ proposta para avaliar a qualidade metodológica dos estudos. Os aspectos avaliados foram: ano de publicação, idioma, desenho e duração do estudo, tamanho da amostra analisada, perdas, método empregado para avaliação do consumo de vitamina $\mathrm{A}$, objetivo da aplicação, média do consumo de vitamina $\mathrm{A}$, índice de adequação do consumo em relação às recomendações nutricionais (IA\%), principais resultados, estatística empregada, variáveis independentes, vieses e variáveis de confusão. Para facilitar a análise e discussão, os dados foram organizados em tabelas, apresentadas nos resultados desta revisão.

\section{RESULTADOS}

A busca bibliográfica resultou em 9.065 artigos, sendo 346 na base SciELO, 814 na base LILACS e 7.905 na base PubMed. Desses artigos, 99 abordavam especificamente o tema em questão, no entanto, foram excluídos os artigos repetidos nas diferentes bases de dados (91). Ao final, foram selecionados oito artigos para esta revisão (Figura 1).
A Tabela I apresenta a descrição dos estudos segundo ano de publicação, desenho, duração, amostra e perdas. Observou-se que a maioria dos estudos $(87,5 \%)$ teve delineamento transversal ${ }^{(15,19-24)}$. Destaca-se que o estudo mais recente foi publicado em $2011^{(25)}$, não havendo relato, de acordo com os descritores utilizados, de publicações sobre a temática nos últimos três anos. Com relação às características da amostra, nota-se que somente quatro estudos $^{(15,21-24)}$ descreveram a faixa etária das participantes. Nos demais, houve relato apenas da média da idade obtida $^{(22,25)}$ ou não houve nenhuma informação sobre essas características $^{(19,20)}$. Apenas um estudo relatou informações sobre as características das perdas do grupo em estudo ${ }^{(24)}$.

Observando a Tabela II, verifica-se que, em quatro artigos, a distribuição das variáveis de confusão não estava descrita no estudo ${ }^{(19,20,23,25)}$, e em apenas dois $\operatorname{artigos}^{(19,21)}$ houve representatividade dos indivíduos incluídos no estudo, portanto, são dados não passíveis de generalização. Dentre os artigos selecionados para esta revisão, todos utilizaram uma estatística aplicada ao objetivo proposto, exceto um estudo ${ }^{(22)}$, cujo método estatístico não foi bem esclarecido.

Segundo a Tabela III, em todos os artigos, os métodos de investigação de consumo alimentar utilizados em gestantes foram R24h e/ou QFA. O método R24h foi utilizado em cinco artigos: três de forma isolada ${ }^{(20,22,23)}$ e dois de forma combinada com o $\mathrm{QFA}^{(21,24)}$. O QFA foi utilizado de forma isolada em três estudos ${ }^{(15,19,25)}$. Quanto ao consumo de vitamina A na população avaliada, um estudo ${ }^{(15)}$ não quantificou a ingestão, apenas verificou a sua frequência. Dentre os outros artigos, somente dois ${ }^{(22,23)}$ calcularam a adequação de ingestão de vitamina $\mathrm{A}$.

Tabela I - Descrição dos estudos sobre consumo de vitamina A segundo ano de publicação, desenho, características da amostra e perdas em gestantes no Brasil, de 1999 a 2015.

\begin{tabular}{|c|c|c|c|c|}
\hline Autores & $\begin{array}{c}\text { Ano da } \\
\text { publicação }\end{array}$ & $\begin{array}{c}\text { Desenho do } \\
\text { estudo }\end{array}$ & Características da amostra inicial & $\begin{array}{c}\text { Perdas do } \\
\text { estudo }\end{array}$ \\
\hline Rondó et al. ${ }^{(19)}$ & 1999 & Transversal & $\begin{array}{l}\text { Gestantes (faixa etária não relatada) - } \\
\qquad \mathrm{n}=710\end{array}$ & Não relatada \\
\hline Saunders et al. ${ }^{(20)}$ & 2000 & Transversal & $\begin{array}{l}\text { Gestantes (faixa etária não relatada) - } \\
\text { n=198 }\end{array}$ & Não relatada \\
\hline Villar e Roncada ${ }^{(21)}$ & 2002 & Transversal & Gestantes (19 a 40 anos $)-\mathrm{n}=91$ & Não relatada \\
\hline Nascimento e Souza ${ }^{(22)}$ & 2002 & Transversal & $\begin{array}{l}\text { Gestantes com sobrepeso (idade média } \\
\text { de } 26 \text { anos) }-\mathrm{n}=110\end{array}$ & Não relatada \\
\hline Azevedo e Sampaio ${ }^{(23)}$ & 2003 & Transversal & $\begin{array}{l}\text { Gestantes adolescentes (14 a } 19 \text { anos) } \\
\qquad-n=91\end{array}$ & Não relatada \\
\hline Giacomello et al. ${ }^{(24)}$ & 2008 & Transversal & Gestantes (15 a 42 anos) $-\mathrm{n}=161$ & 9 perdas \\
\hline Santos et al. ${ }^{(15)}$ & 2010 & Transversal & Gestantes (18-35 anos) $-\mathrm{n}=92$ & Não relatada \\
\hline Fazio et al. ${ }^{(25)}$ & 2011 & Retrospectivo & $\begin{array}{l}\text { Gestantes (idade média de 30,6 anos) - } \\
\qquad \mathrm{n}=197\end{array}$ & Não relatada \\
\hline
\end{tabular}


Tabela II - Aspectos metodológicos e científicos dos estudos sobre consumo de vitamina A em gestantes no Brasil, de 1999 a 2015.

\begin{tabular}{|c|c|c|c|c|c|}
\hline Autores & $\begin{array}{c}\text { Ano da } \\
\text { publicação }\end{array}$ & $\begin{array}{c}\text { Variáveis } \\
\text { independentes }\end{array}$ & $\begin{array}{c}\text { Viés/ Variáveis de } \\
\text { confusão }\end{array}$ & Principais resultados & Estatística empregada \\
\hline $\begin{array}{l}\text { Rondó et } \\
\text { al. }^{(19)}\end{array}$ & 1999 & - & - & $\begin{array}{l}49,5 \% \text { das gestantes } \\
\text { estavam com baixo } \\
\text { risco para deficiência } \\
\text { de vitamina A e } 50,5 \% \\
\text { estavam em risco } \\
\text { moderado }\end{array}$ & $\begin{array}{l}\text { - Análise de variância } \\
\text { - Coeficiente de } \\
\text { correlação de Spearman }\end{array}$ \\
\hline $\begin{array}{l}\text { Saunders et } \\
\text { al. }^{(20)}\end{array}$ & 2000 & - & - & $\begin{array}{l}\text { Constatou-se grande } \\
\text { flutuação dos valores } \\
\text { médios de vitamina A }\end{array}$ & $\begin{array}{l}\text { - Análise da variância } \\
\text { - Teste de Tukey }\end{array}$ \\
\hline $\begin{array}{c}\text { Villar e } \\
\text { Roncada }^{(21)}\end{array}$ & 2002 & $\begin{array}{l}\text { Período de } \\
\text { gestação, nível } \\
\text { de escolaridade, } \\
\text { ocupação }\end{array}$ & $\begin{array}{l}\text { Sazonalidade, } \\
\text { hábitos alimentares } \\
\text { e diferentes } \\
\text { trimestres da } \\
\text { gestação }\end{array}$ & $\begin{array}{l}55 \% \text { das gestantes } \\
\text { apresentaram consumo } \\
\text { médio de vitamina A } \\
\text { acima das recomendações }\end{array}$ & $\begin{array}{l}\text { - Análise de variância } \\
\text { - Coeficiente de } \\
\text { correlação de Spearman } \\
\text { - Teste de Kappa }\end{array}$ \\
\hline $\begin{array}{l}\text { Nascimento } \\
\text { e Souza }^{(22)}\end{array}$ & 2002 & $\begin{array}{l}\text { Idade, nível de } \\
\text { escolaridade, } \\
\text { renda, número } \\
\text { de gestações } \\
\text { anteriores, peso, } \\
\text { altura, índice de } \\
\text { massa corpórea }\end{array}$ & $\begin{array}{l}\text { Orientação } \\
\text { nutricional das } \\
\text { gestantes antes da } \\
\text { coleta de dados }\end{array}$ & $\begin{array}{l}\text { A dieta mostrou-se } \\
\text { satisfatória em energia } \\
\text { e em carboidratos. } \\
\text { Proteínas, lipídios } \\
\text { e vitaminas A e } \\
\text { C atingiram e/ } \\
\text { ou ultrapassaram } \\
\text { as quantidades } \\
\text { recomendadas, enquanto } \\
\text { que cálcio, ferro, e } \\
\text { folato apresentaram o } \\
\text { percentual de adequação } \\
\text { de } 50 \%, 33 \% \text {, e } 43 \%, \\
\text { respectivamente }\end{array}$ & $\begin{array}{l}\text { - Teste de diferença de } \\
\text { médias (?) }\end{array}$ \\
\hline $\begin{array}{l}\text { Azevedo e } \\
\text { Sampaio }^{(23)}\end{array}$ & 2003 & $\begin{array}{l}\text { Idade, renda } \\
\text { familiar, } \\
\text { escolaridade, } \\
\text { condições de } \\
\text { moradia, idade } \\
\text { gestacional, } \\
\text { peso, altura, } \\
\text { índice de massa } \\
\text { corpórea }\end{array}$ & - & $\begin{array}{l}\text { O consumo de vitamina A } \\
\text { e C atingiu o percentual } \\
\text { de } 100 \% \text { em } 58,2 \% \text { e } \\
53,3 \% \text { das gestantes, } \\
\text { respectivamente. } 63,6 \% \\
\text { das dietas tinham } \\
\text { menos que } 90 \% \text { das } \\
\text { necessidades energéticas } \\
\text { e mais da metade estava } \\
\text { adequada em proteínas, } \\
\text { com excesso de lipídios } \\
\text { e conteúdo inadequado } \\
\text { de carboidratos. Ferro, } \\
\text { cálcio, ácido fólico, } \\
\text { vitamina B } 1 \text { e zinco, } \\
\text { foram consumidos em } \\
\text { quantidade bem abaixo } \\
\text { do recomendado }\end{array}$ & $\begin{array}{l}\text { Distribuição de } \\
\text { frequências }\end{array}$ \\
\hline
\end{tabular}




\begin{tabular}{|c|c|c|c|c|c|}
\hline $\begin{array}{l}\text { Giacomello } \\
\text { et al. }{ }^{(24)}\end{array}$ & 2008 & $\begin{array}{l}\text { Situação } \\
\text { conjugal, nível } \\
\text { de ocupação, } \\
\text { escolaridade, } \\
\text { orientação } \\
\text { nutricional, } \\
\text { faixa etária, } \\
\text { idade } \\
\text { gestacional, no } \\
\text { de pessoas em } \\
\text { casa, renda per } \\
\text { capita }\end{array}$ & $\begin{array}{l}\text { Escolaridade, } \\
\text { condição } \\
\text { socioeconômica, } \\
\text { curto intervalo } \\
\text { de tempo para a } \\
\text { coleta de dados e } \\
\text { ausência de medidas } \\
\text { que permitam } \\
\text { quantificar o viés } \\
\text { entrevistador e } \\
\text { entrevistado }\end{array}$ & $\begin{array}{l}\text { O QFA superestimou } \\
\text { o consumo de energia } \\
\text { e nutrientes. Foram } \\
\text { observadas baixas } \\
\text { correlações de vitaminas } \\
\text { A, e C e maiores } \\
\text { correlações para as fibras, } \\
\text { vitamina C, ácido fólico, } \\
\text { cálcio e potássio }\end{array}$ & $\begin{array}{l}\text { - Teste de comparação } \\
\text { de médias/ variáveis } \\
\text { (Teste T e quiquadrado) } \\
\text { - Teste de concordância } \\
\text { e correlação de } \\
\text { métodos: LOAS, } \\
\text { Kappa, coeficiente de } \\
\text { correlação de Pearson } \\
\text { bruto e ajustado }\end{array}$ \\
\hline $\begin{array}{l}\text { Santos et } \\
\text { al. }^{(15)}\end{array}$ & 2010 & $\begin{array}{l}\text { Idade, renda } \\
\text { per capita, } \\
\text { escolaridade, } \\
\text { estado } \\
\text { civil, estado } \\
\text { nutricional, } \\
\text { ocorrência de } \\
\text { abortos, } \mathrm{n}^{\circ} \\
\text { de gestações, } \\
\text { intercorrências }\end{array}$ & $\begin{array}{l}\text { Condição } \\
\text { socioeconômica e } \\
\text { hábitos alimentares }\end{array}$ & $\begin{array}{l}\text { Observou-se menor } \\
\text { consumo de vitamina } \\
\text { A pelas gestantes com } \\
\text { cegueira noturna }\end{array}$ & $\begin{array}{l}\text { - Teste exato de Fisher } \\
\text { - Teste de Wilcoxon }\end{array}$ \\
\hline Fazio et al. ${ }^{(25)}$ & 2011 & $\begin{array}{l}\text { Peso, altura, } \\
\text { índice de massa } \\
\text { corpórea }\end{array}$ & - & $\begin{array}{l}\text { As gestantes de baixo } \\
\text { peso apresentaram menor } \\
\text { consumo de lipídios } \\
\text { quando comparadas às } \\
\text { eutróficas. A ingestão } \\
\text { diária de vitamina A, } \\
\text { vitamina C, e cálcio } \\
\text { foi semelhante entre } \\
\text { os grupos. A média de } \\
\text { ingestão de ferro e folato } \\
\text { foi maior em gestantes } \\
\text { eutróficas, quando } \\
\text { comparadas às com } \\
\text { sobrepeso e baixo peso }\end{array}$ & $\begin{array}{l}\text { - Distribuição de } \\
\text { frequência } \\
\text {-Teste quiquadrado }\end{array}$ \\
\hline
\end{tabular}


Tabela III - Métodos de investigação do consumo alimentar, objetivo da aplicação do método, média de consumo de vitamina A e índice de adequação (IA\%).

\begin{tabular}{|c|c|c|c|c|c|}
\hline Autores & $\begin{array}{c}\text { Ano da } \\
\text { publicação }\end{array}$ & $\begin{array}{c}\text { Método de } \\
\text { investigação do } \\
\text { consumo alimentar }\end{array}$ & $\begin{array}{c}\text { Objetivo da aplicação do } \\
\text { método }\end{array}$ & $\begin{array}{l}\text { Consumo médio de } \\
\text { vitamina A ( } \mu \text { gRE) }\end{array}$ & IA (\%) \\
\hline $\begin{array}{l}\text { Rondó et } \\
\text { al. }^{(19)}\end{array}$ & 1999 & 1 QFA* & $\begin{array}{c}\text { Analisar o risco da DVA*** } \\
\text { em gestantes }\end{array}$ & 487,10 & - \\
\hline $\begin{array}{l}\text { Saunders et } \\
\text { al. }{ }^{(20)}\end{array}$ & 2000 & $1 \mathrm{R} 24 \mathrm{~h} * *$ & $\begin{array}{c}\text { Verificar a flutuação do } \\
\text { conteúdo médio de Vit. A } \\
\text { das dietas de gestantes, com } \\
\text { base em } 5 \text { diferentes tabelas } \\
\text { de composição química dos } \\
\text { alimentos }\end{array}$ & $\begin{array}{c}\text { 1008,89 (INCAP****, } \\
1961 \text { ) } \\
\text { 1204,90 } \\
\text { (FIBGE*****, 1996) } \\
\text { 1494,06 (Tabela do } \\
\text { Grupo de Pesquisa } \\
\text { em Nutrição Materno- } \\
\text { infantil da UFRJ, 1996) } \\
\text { 793,02 (Tabela de } \\
\text { Franco, 1992) } \\
\text { 908,97 (Tabela } \\
\text { Handbook, (1993) }\end{array}$ & - \\
\hline $\begin{array}{c}\text { Villar e } \\
\text { Roncada }^{(21)}\end{array}$ & 2002 & $\begin{array}{l}1 \text { Formulário dietético } \\
\text { simplificado }\end{array}$ & $\begin{array}{l}\text { Identificar o consumo de } \\
\text { alimentos fontes de Vit. A } \\
\text { em gestantes }\end{array}$ & 1134,00 & - \\
\hline $\begin{array}{l}\text { Nascimento } \\
\text { e Souza }\end{array}$ & 2002 & $1 \mathrm{R} 24 \mathrm{~h}$ & $\begin{array}{l}\text { Verificar o valor energético } \\
\text { e a adequação nutricional de } \\
\text { dietas de gestantes }\end{array}$ & 1059,40 & $132,43 \%$ \\
\hline $\begin{array}{l}\text { Azevedo e } \\
\text { Sampaio }^{(23)}\end{array}$ & 2003 & $\begin{array}{l}\mathrm{R} 24 \mathrm{~h}-3 \text { dias não } \\
\text { consecutivos }\end{array}$ & $\begin{array}{l}\text { Analisar o consumo } \\
\text { alimentar de gestantes }\end{array}$ & 820,80 & $58,2 \%$ \\
\hline $\begin{array}{l}\text { Giacomello } \\
\text { et al. }{ }^{(24)}\end{array}$ & 2008 & $\begin{array}{c}1 \text { QFA - validado; } \\
\text { R24h ( } 2 \text { para gestantes } \\
\text { que realizaram } 1 \\
\text { entrevista e } 4 \text { para } \\
\text { as que realizaram } 2 \\
\text { entrevistas em dias não } \\
\text { consecutivos) }\end{array}$ & $\begin{array}{l}\text { Avaliar o desempenho do } \\
\text { questionário de frequência } \\
\text { alimentar em medir o } \\
\text { consumo alimentar de } \\
\text { gestantes }\end{array}$ & $\begin{array}{l}\mathrm{R} 24 \mathrm{~h}=927,00 \\
\mathrm{QFA}=1601,00\end{array}$ & - \\
\hline $\begin{array}{l}\text { Santos et } \\
\text { al. } .^{(15)}\end{array}$ & 2010 & 1 QFA (qualitativo) & $\begin{array}{l}\text { Analisar a frequência do } \\
\text { consumo de alimentos fontes } \\
\text { de Vit. A em gestantes }\end{array}$ & Não avaliado & - \\
\hline $\begin{array}{l}\text { Fazio et } \\
\text { al. }{ }^{(25)}\end{array}$ & 2011 & $1 \mathrm{QFA}$ & $\begin{array}{c}\text { Avaliar a ingestão de macro } \\
\text { e micronutrientes, de acordo } \\
\text { com o estado nutricional } \\
\text { materno }\end{array}$ & $\begin{array}{c}\text { Baixo peso }=693,70 \\
\text { Eutrofia }=703,00 \\
\text { Sobrepeso }=666,10 \\
\text { Obesidade }=519,8\end{array}$ & - \\
\hline
\end{tabular}

*QFA: Questionário de frequência de consumo alimentar;

**R24h: Recordatório de 24 horas;

***DVA: Deficiência de vitamina A; Vit. A: Vitamina A;

****Instituto de Nutrición Centro America y Panamá

*****Fundação Instituto Brasileiro de Geografia e Estatística 
Descritores utilizados: deficiência de vitamina $\mathrm{A}$, consumo alimentar, alimentação, ingestão de alimentos, vitamina $\mathrm{A}$, gestantes e gravidez e seus correspondentes em inglês e espanhol

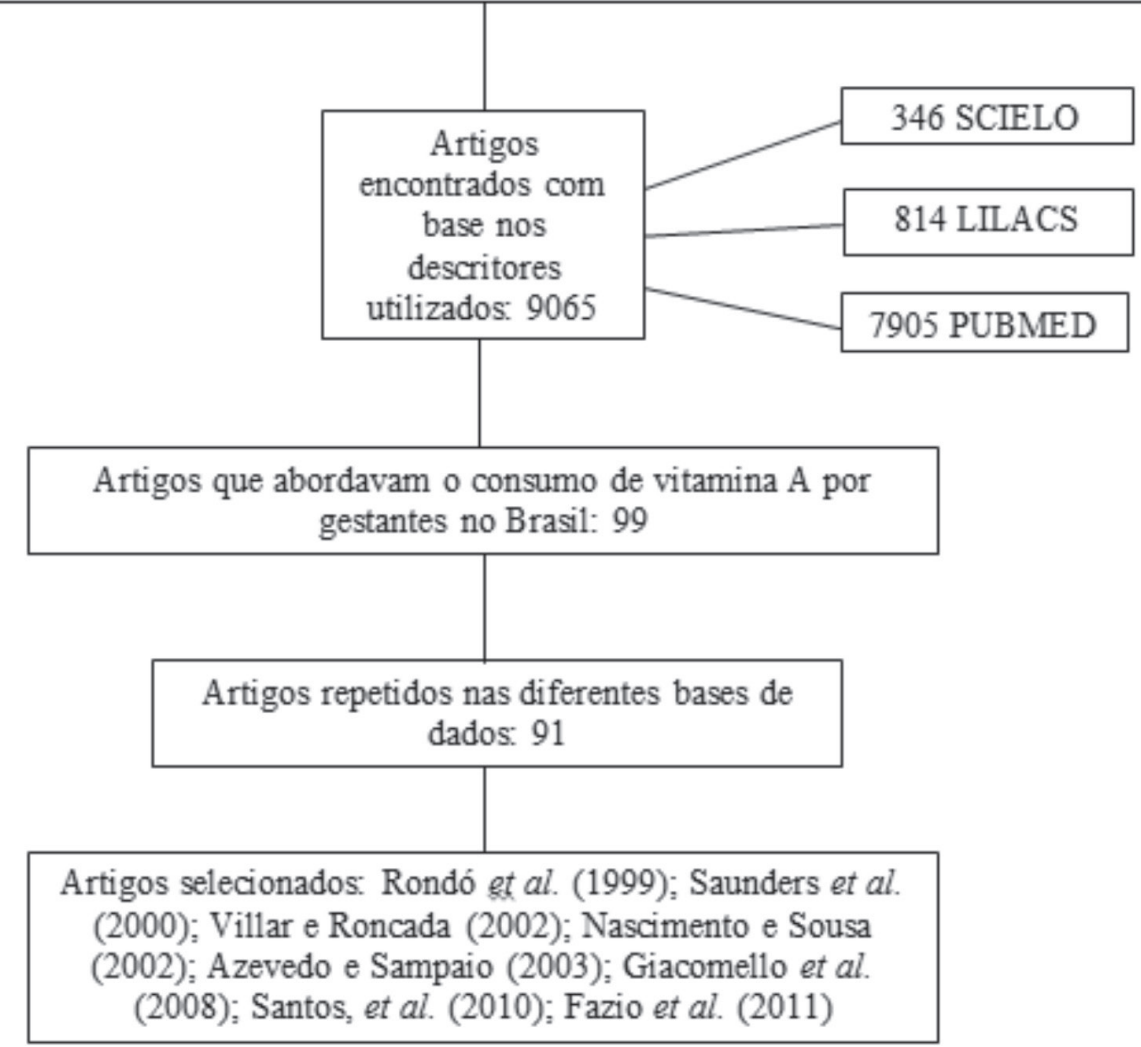

Figura 1 - Fluxograma dos artigos selecionados.

\section{DISCUSSÃO}

Várias metodologias vêm sendo empregadas para investigar o consumo alimentar em gestantes, dentre elas, os métodos que mais se destacam são o R24h e o QFA. Entretanto, ressalta-se que, devido ao uso inadequado e às limitações de cada método, aliado à escassez de instrumentos validados e reprodutíveis, ainda são poucos os estudos que refletem de forma fidedigna a ingestão alimentar na gestação ${ }^{(26)}$.

Destaca-se que, entre os artigos que buscaram avaliar o consumo de vitamina A por gestantes brasileiras, no que se refere ao delineamento metodológico, a maioria (sete) dos estudos utilizou desenho transversal, porém, com período de coleta de dados muito extenso, superando três meses em cinco artigos. Se considerarmos o efeito da sazonalidade na disponibilidade de alimentos fontes de vitamina A no Brasil, o aspecto do curto período de coleta de informação sobre consumo alimentar ganha uma importância ainda maior, uma vez que a disponibilidade de alimentos pode se modificar consideravelmente nas diferentes estações do $\mathrm{ano}^{(27)}$.

Ao contrário da maioria dos estudos, somente $u^{(25)}$ realizou um estudo de caráter retrospectivo. Os estudos retrospectivos são chamados de recordatórios ou de caso-controle, são mais baratos e rápidos que os estudos prospectivos, porém, menos confiáveis, pois embora gastem menos tempo e recursos, eles se fundamentam na capacidade de recordar dos informantes, tornando menos exata a quantificação dos fatores de risco e a determinação da sequência temporal entre a exposição ao fator e o aparecimento do desfecho que se deseja avaliar ${ }^{(28)}$.

No tocante aos métodos de seleção da amostra de gestantes dos estudos aqui analisados, observa-se que obedeceram ao critério de conveniência. As amostras de conveniência são chamadas de não aleatórias ou não 
probabilísticas, significando que os elementos que dela fazem parte foram selecionados por um julgamento de valor, e não por questões de aleatoriedade estatística. Ou seja, foram incluídos no estudo os indivíduos de mais fácil acesso $^{(29)}$, tendo em vista o fato de que os únicos estudos que citaram ter realizado cálculo amostral foram somente dois $^{(19,21)}$.

O uso de dados de rotina derivados do atendimento da demanda nos serviços de saúde, cujos resultados são extrapolados para toda população, havendo escolha não aleatória dos membros para compor a amostra da investigação populacional, levam a suspeitar da presença do viés de seleção em uma investigação ${ }^{(29)}$.

Somente em um estudo ${ }^{(24)}$ as perdas foram relatadas, mas não foram superiores a $6 \%$ da amostra inicial. Ressalta-se que a ausência da quantificação das perdas pode comprometer as estimativas de associação de estudos, como também a não realização do controle de alguns importantes fatores de confusão, sendo estes capazes de interferir negativamente nos resultados ${ }^{(30)}$.

Entre os estudos analisados, seis controlaram as variáveis independentes, sendo as mais citadas: escolaridade, idade, renda, peso, altura e índice de massa corpórea (IMC). Destaca-se que as variáveis independentes servem para expor a situação de saúde de subgrupos da população, fornecer subsídios para explicações causais, ou para levantamento de hipóteses, que constituem o ponto de partida para outros estudos, além de definir prioridades de intervenção, de modo a influenciar a direção das medidas de prevenção e controle ${ }^{(29)}$.

Dentre as variáveis independentes citadas como fatores de confusão pelos $\operatorname{artigos}^{(19,20,23,25)}$, está a escolaridade das gestantes, pois pode influenciar o relato do consumo por meio do QFA, e a condição socioeconômica menos privilegiada, que pode gerar constrangimento, fazendo com que as gestantes superestimem o seu consumo. Além destas, também foram citadas como possíveis fatores a sazonalidade, os hábitos alimentares e a não aplicação dos inquéritos alimentares nos diferentes trimestres da gestação. Este último é de suma importância, pois a formação de reservas fetais de vitamina $\mathrm{A}$ se inicia durante o último trimestre de gestação e, após o nascimento, necessita de vários meses de ingestão adequada para construir suas reservas $^{(31,32)}$.

Em um dos artigos, foi considerado como um possível viés o fato de gestantes com sobrepeso terem recebido orientação nutricional antes da coleta de dados, o que pode ter ocasionado a subnotificação da ingestão de alimentos.

Muitos estudos que utilizam os inquéritos alimentares não consideram fatores importantes, tais como viés de memória, sobretudo quanto às informações coletadas retrospectivamente, como é o caso do R24h e do QFA. Além disso, outros fatores podem dificultar o registro da ingestão alimentar dos indivíduos, como complexidade da dieta, hábitos alimentares, qualidade da informação, idade, imagem corporal, memória do entrevistado, crenças, comportamento, cultura e status socioeconômico ${ }^{(33)}$. É imprescindível conhecer as limitações de cada método, já que estes constituem potenciais fontes de vieses. Então, quando bem conduzidos, os estudos podem fornecer dados precisos do consumo alimentar, contanto que os pesquisadores estejam bem treinados, e os entrevistados, motivados $^{(17,26)}$.

Antes da publicação da Dietary Reference Intakes $\mathrm{DRI}^{(34)}$, utilizava-se a Recommended Dietary Allowance - $\mathrm{RD}^{(35)}$ para categorização da inadequação de consumo, tanto para indivíduos quanto para estudos populacionais. Entretanto, atualmente, a estimativa de referência apropriada para a avaliação da inadequação da ingestão de nutrientes é a EAR (Estimated Averagere Requirement), definida como o valor de ingestão do nutriente que corresponde à necessidade média estimada para determinado estágio de vida e gênero. $\mathrm{O}$ valor da EAR da vitamina A para gestantes adultas é $550 \mu \mathrm{gER}$, e para gestantes adolescentes é de 530 $\mu \mathrm{gER}^{(36)}$.

O consumo de vitamina A pelas gestantes no pósparto foi investigado através de um questionário de frequência alimentar simplificado, não validado, referente ao consumo habitual durante os nove meses de gravide $z^{(19)}$. A categorização das mulheres em alto, moderado e baixo risco para deficiência de consumo de vitamina A foi feita tomando como base as recomendações da $\mathrm{RDA}^{(35)}$, que preconiza o consumo de $800 \mu \mathrm{gER} /$ dia. O valor médio de ingestão de vitamina A foi 487,31 $\mu \mathrm{gER} /$ dia. Apesar de o estudo ter concluído que a maioria das mulheres não tinha um alto risco para DVA, estas afirmaram que a aplicação do questionário de frequência alimentar não é suficiente para avaliar o status da vitamina $\mathrm{A}$, provavelmente devido à variação do consumo.

Em outra pesquisa, foi verificada flutuação do conteúdo médio de vitamina $\mathrm{A}$ das dietas, com base em cinco diferentes tabelas, através de inquérito $R 24 \mathrm{~h}^{(20)}$. Os valores médios de vitamina $\mathrm{A}$ das dietas, a partir das cinco tabelas, foram: 1008,89 $\mu \mathrm{gER}^{(37)} ; 1204,90 \mu \mathrm{gER}^{(38)}$; $1494,06 \mu \mathrm{gER}^{(39)} ; 793,02 \mu \mathrm{gER}^{(40)}$ e $908,97 \mu \mathrm{gER}^{(41)}$. Foi observada grande flutuação dos valores médios de vitamina A, além de encontrarem-se acima da recomendação de ingestão sugerida pela $\mathrm{FAO}^{(42)}$, que é de $600 \mu \mathrm{gER} / \mathrm{dia}$, utilizada pelos autores na avaliação da adequação dietética.

Apesar de os autores supracitados terem considerado uma criteriosa metodologia para aplicação do recordatório, como utilização de medidas caseiras, figuras representativas de tamanho de porções de alimentos, e treinamento do 
entrevistador, a aplicação de um único recordatório não é capaz de representar a ingestão habitual de um indivíduo. Além disso, dentre todas as limitações desse inquérito, a mais preocupante é que ele não fornece uma estimativa segura da absorção de nutrientes, devido à variação do dia a dia; e quando usado em estudos epidemiológicos, a habilidade de descrever relações significantes entre dieta e risco de doença fica reduzida drasticamente ${ }^{(33)}$.

Ressalta-se, ainda, que a identificação e controle dos possíveis vieses do inquérito dietético poderão melhorar sua fidedignidade como indicador precoce e pré-patológico do estado nutricional da vitamina $\mathrm{A}^{(20)}$.

Um dos estudos selecionados ${ }^{(21)}$ avaliou o consumo de vitamina A por meio do formulário dietético simplificado, composto por um questionário R24h (aplicado por um entrevistador treinado, mas não há relatos quanto à padronização de medidas caseiras) e um QFA. Em outro(22), verificou-se o valor energético e a adequação nutricional da ingestão dietética de gestantes com sobrepeso prévio ou adquirido durante a gestação, observando-se a ocorrência de consumo adequado de nutrientes. Para investigação do consumo alimentar, foi empregado apenas um R24h, e não foi relatado se houve padronização de medidas caseiras e se o entrevistador era treinado. A recomendação dietética utilizada foi a $\mathrm{RDA}^{(35)}$, observando-se um percentual de $132,43 \%$ de adequação de vitamina A. No entanto, acredita-se que o consumo alimentar foi subestimado pelas gestantes, já que elas receberam orientação nutricional antes da coletas dos dados.

O consumo alimentar em gestantes adolescentes atendidas em um serviço público de referência para assistência pré-natal em Fortaleza também foi investigado $^{(23)}$. O método utilizado foi o R24h aplicado em três dias não consecutivos. Não foi relatado se houve padronização das medidas caseiras ou treinamento da equipe. Observou-se que $58,2 \%$ das gestantes apresentaram um consumo de vitamina A adequado, de acordo com a $\mathrm{DRI}^{(34)}$, cuja recomendação para gestante adolescente é de $770 \mu \mathrm{gER} /$ dia. No entanto, apesar de os autores terem referido essa recomendação, pode-se perceber, através da análise dos resultados, que a referência realmente utilizada para o cálculo da adequação foi a $\mathrm{RDA}^{(35)}$, que preconiza $800 \mu \mathrm{gRE} / \mathrm{dia}$.

Em estudo com gestantes usuárias dos SUS de Porto Alegre e Bento Gonçalves, foram aplicados dois recordatórios para quem realizou uma entrevista, e quatro para as que realizaram duas entrevistas ${ }^{(24)}$. Realizaram-se os recordatórios através de formulário padronizado, e os entrevistadores eram treinados. Utilizou-se questionário de frequência alimentar validado ${ }^{(43)}$, composto por oitenta alimentos. Com a aplicação dos recordatórios, a média de consumo de vitamina A foi de $927 \mu \mathrm{gER} / \mathrm{dia}$, e com o questionário de frequência, foi de $1601 \mu \mathrm{gER} /$ dia. Os autores observaram que houve uma superestimação de energia e dos outros nutrientes pelo questionário de frequência alimentar.

A associação da cegueira noturna com as variáveis nutricionais em gestantes também foi avaliada ${ }^{(15)}$. Para isso, aplicou-se um questionário de frequência alimentar qualitativo, não validado, composto por 23 alimentos. Por ser qualitativo, ele não trouxe a média de ingestão de vitamina A, porém, os autores observaram um menor consumo de vitamina A pelas gestantes com cegueira noturna.

Quanto à estatística dos artigos analisados, a maioria deles (sete) descreveu o método estatístico utilizado, exceto $u^{(22)}$, que não identificou o teste de diferença de média aplicado e realizou uma análise nos resultados cujo método estatístico empregado não foi relatado.

Por fim, ressalta-se que o uso de diferentes tabelas de composição de alimentos, algumas sem uma adequada informação do conteúdo de alimentos regionais, pode ter contribuído para a ampla variação no consumo médio de vitamina A entre os estudos revisados. Sendo assim, nota-se que há necessidade de mais estudos criteriosos quanto aos seus aspectos metodológicos, para que as possíveis fontes de erros e vieses sejam minimizadas através de instrumentos validados e reprodutíveis.

\section{CONCLUSÃO}

Observa-se que ainda são escassos estudos que avaliem de forma criteriosa o consumo de vitamina A por gestantes no Brasil, e que a identificação e controle dos possíveis vieses do inquérito dietético poderão melhorar a fidedignidade dos dados encontrados.

\section{CONFLITOS DE INTERESSE}

Os autores declaram não haver conflitos de interesse.

\section{REFERÊNCIAS}

1. Graebner IT, Saito CH, Souza EMD. Avaliação bioquímica de vitamina A em escolares de uma comunidade rural. J Pediatr (Rio J). 2007;83(3):24752.

2. World Health Organization - WHO. Global prevalence of vitamin A deficiency in populations at risk 19952005: WHO global database on vitamin A deficiency. Geneva: WHO; 2009.

3. Tuma MAF, Roncada MJ, César TB. Adequação na ingestão de vitamina A por gestantes, segundo 
as Dietary Reference Intakes. CuidArte Enferm. 2014;8(1):9-15.

4. Miglioli TC, Fonseca VM, Gomes Junior SC, Lira PICD, Batista-Filho M. Deficiência de Vitamina A em mães e filhos no Estado de Pernambuco. Ciênc Saúde Coletiva. 2013;18(5):1427-40.

5. Ministério da Saúde (BR). Relatório Final da Pesquisa Nacional de Demografia e Saúde. Brasília: Pesquisa Nacional de Demografia e Saúde da Criança e da Mulher; 2006. [acesso em 2015 Jul 30]. Disponível em: http://www. saude.gov.br/pnds2006

6. Paiva AA, Rondó PH, Vaz-de-Lima LR, Oliveira CF, Ueda M, Goncalves-Carvalho C, et al. The impact of vitamin A supplementation on the immune system of vitamin A-deficient children. Int J Vitam Nutr Res. 2010;80(3):188-96.

7. Kositamongkol S, Suthutvoravut U, Chongviriyaphan N, Feungpean B, Nuntnarumit P. Vitamin A and E status in very low birth weight infants. J Perinatol. 2011;31(7):471-6.

8. Pedraza DF. Baixo peso ao nascer no Brasil: revisão sistemática de estudos baseados no sistema de informações sobre nascidos vivos. Rev Atenção Saúde. 2014;12(41):37-50.

9. Saraiva BC, Soares MC, Santos LCD, Pereira SC, Horta PM. Iron deficiency and anemia are associated with low retinol levels in children aged 1 to 5 years. J Pediatr (Rio J). 2014;90(6):593-9.

10. Huang Y, Zheng S. The effect of vitamin A deficiency during pregnancy on anorectal malformations. J Pediatr Surg. 2011;46(7):1400-5.

11. Campos LF, Saunders C, Ramalho A, Gomes MM, Accioly E. Níveis de retinol e carotenóides séricos e intercorrências gestacionais em puérperas. Rev Nutr. 2008;21(6):623-32.

12. Van Den Broek N, Dou L, Othman M, Neilson JP, Gates S, Guelmezoglu AM. Vitamin A supplementation during pregnancy for maternal and newborn outcomes. Cochrane Database Syst Rev. 2010;(11):CD008666.

13. West KP, Christian P, Labrique AB, Rashid M, Shamim AA, Klemm RD, et al. Effects of vitamin A or beta carotene supplementation on pregnancy-related mortality and infant mortality in rural Bangladesh: a cluster randomized trial. Jama. 2011;305(19):1986-95.

14. Masanja H, Smith ER, Muhihi A, Briegleb C, Mshamu S, Ruben J, et al. Effect of neonatal vitamin A supplementation on mortality in infants in Tanzania
(Neovita): a randomised, double-blind, placebocontrolled trial. Lancet. 2015;385(9975):1324-32.

15. Santos EM, Velardes LGC, Ferreira VA. Associação entre deficiência de vitamina $A$ e variáveis socioeconômicas, nutricionais e obstétricas de gestantes. Ciênc Saúde Coletiva 2010;15(1):1021-30.

16. Anjos LAD, Souza DRD, Rossato SL. Desafios na medição quantitativa da ingestão alimentar em estudos populacionais. Rev Nutr. 2009;22(1):151-61.

17. Bertin RL, Parisenti J, Di Pietro PF, Vasconcelos FDAGD. Métodos de avaliação do consumo alimentar de gestantes: uma revisão. Rev Bras Saúde Matern Infant. 2006;6(4):383-90.

18. Downs SH, Black N. The feasibility of creating a checklist for the assessment of the methodological quality both of randomised and non-randomised studies of health care interventions. J Epidemiol Community Health 1998;52(6):377-84

19. Rondó PHE, Villar BS, Tomkins AM. Vitamin A status of pregnant women assessed by a biochemical indicator and a simplified food frequency questionnaire. Arch Latinoam Nutr 1999;49(4):322-5.

20. Saunders C, Ramalho A, Accioly E, Paiva F. Utilização de tabelas de composição de alimentos na avaliação do risco de hipovitaminose A. Arch Latinoam Nutr. 2000;50(3)237-42.

21. Villar BS, Roncada MJ. Determinação do consumo de alimentos fontes de vitamina A por gestantes, utilizando o Formulário Dietético Simplificado (FDS). Arch Latinoam Nutr 2002;52(1):48-54.

22. Nascimento E, Souza SB. Avaliação da dieta de gestantes com sobrepeso. Rev Nutr 2002;15(2):174-79.

23. Azevedo DV, Sampaio HAC. Consumo alimentar de gestantes adolescentes atendidas em serviço de assistência pré- natal. Rev Nutr. 2003;16(3):273-80.

24. Giacomello A, Schmidt MI, Nunes MAA, Duncan BB, Soares RM, Manzolli P, et al. Validação relativa do questionário de frequência alimentar em gestantes usuárias do Serviço Único de Saúde em dois municípios do Rio Grande do Sul, Brasil. Rev Bras Saúde Matern Infant. 2008;8(4):445-54.

25. Fazio ES, Nomura RMY, Dias MCG, Zugab $M$. Consumo dietético de gestantes e ganho ponderal materno após aconselhamento nutricional. Rev Bras Ginecol Obstet. 2011;33(2):87-92.

26. Sartorelli DS, Barbieri P. Número de replicações de inquéritos dietéticos para estimativa da ingestão de 
nutrientes em gestantes brasileiras. Rev Bras Saúde Matern Infant. 2014;14(4):441-5.

27. Costa AF, Yokoo EM, Anjos LAD, WahrlichI V, Olinto MTA, Henn RL, Waissmann W. Variação sazonal na ingestão alimentar de adultos de Niterói. Rev Bras Epidemiol. 2013;16(2):513-24.

28. Taddei JAAC, Lang RMF, Longo SG, Toloni MHA. Nutrição em Saúde Pública. Rio de Janeiro: Rubio; 2011.

29. Pereira MG. Epidemiologia: teoria e prática. $6^{\mathrm{a}}$ ed. Rio de Janeiro: Guanabara Koogan; 2002.

30. Araújo DMR, Pereira NL, Kac G. Ansiedade na gestação, prematuridade e baixo peso ao nascer: uma revisão sistemática da literatura. Cad Saúde Pública 2007;23(4):747-56.

31. Silva LDSVD, Thiapó AP, Souza GGD, Saunders C, Ramalho A. Micronutrientes na gestação e lactação. Rev Bras Saúde Matern Infant. 2007;7(3):237-44.

32. Pedraza DF, Queiroz DD. Micronutrientes no crescimento e desenvolvimento infantil. Rev Bras Crescimento Desenvolv Hum. 2011;21(1):156-71.

33. Cavalcante AAM, Priore SE, Franceschini SDCC. Estudos de consumo alimentar: aspectos metodológicos gerais e o seu emprego na avaliação de crianças e adolescentes. Rev Bras Saúde Mater Infant. 2004;4(3):229-40.

34. Institute of Medicine - IOM. Dietary reference intakes for vitamin A, vitamin $\mathrm{K}$, arsenic, boron, chromium, copper, iodine, iron, manganese, molybdenum, nickel, silicon, vanadium, andzinc. Washington (DC): National Academy Press; 2002.

35. National Research Council - NRC. Recommended dietary allowances (RDA). $10^{\mathrm{a}}$ ed. Washington (DC): National Academy Press; 1989.

36. Padovani RM, Amaya-Farfán J, Colugnati FAB, Domene SMÁ. Dietary reference intakes: aplicabilidade das tabelas em estudos nutricionais. Rev Nutr. 2006;19(6):741-60.
37. Instituto de Nutrición de Centro américa y Panamá INCAP. Tabla de composición de alimentos para uso en América Latina.Guatemala: INCAP; 1961.

38. Fundação Instituto Brasileiro de Geografia e Estatística - FIBGE. Estudo Nacional da Despesa Familiar. Tabelas de composição dos alimentos. $4^{\mathrm{a}}$ ed. Rio de Janeiro: FIBGE; 1996.

39. Pinheiro ABV, Lacerda EMA, Benzecry EH, Gomes MCS, Costa VM. Tabela para avaliação de consumo alimentar em medidas caseiras. $3^{\mathrm{a}}$ ed. Rio de Janeiro: Grupo de Pesquisa em Nutrição Materno-infantil da UFRJ; 1996.

40. Franco G. Tabela de composição química dos alimentos. $9^{\mathrm{a}}$ ed. Rio de Janeiro: Atheneu; 1992.

41. United States Departament of Agriculture - USDA, Centro de Informática em Saúde da Escola Paulista de Medicina (CIS-EPM). Programa de apoio à nutrição. São Paulo: CIS-EPM; 1993.

42. Food and Agriculture Organization of the United Nations - FAO/OMS. Necessidades de vitamina A, hierro, folato y vitamina B12. Roma: FAO/OMS; 1991.

43. Sichieri R, Everhart JE. Validity of a Brazilian food frequency questionnaire against dietary recalls and estimated energy intake. Nutr Res. 1998;18(10):164959.

\section{Endereço para correspondência:}

Laís Spíndola Garcêz

Universidade Federal do Piauí-UFPI

Campus Ministro Petrônio Portella, s/n

Bairro: Ininga

CEP: 64049-550 - Teresina - PI - Brasil

E-mail: laisspindolagarcez@hotmail.com 